\title{
Research Article \\ Multilevel segmental femur fracture in young individuals treated by a single step - All in one intramedullary device - A prospective study
}

\author{
Senthil Loganathan, U. Thiyagarajan \\ Department of Orthopaedics, Sri Ramachandra Institute for Higher Education and Research \\ Porur, Chennai - 600116. Tamil Nadu INDIA
}

(Received: September 2020

Revised: October 2020

Accepted: November 2020)

Corresponding author: U. Thiyagarajan. Email: dr.thyaga@gmail.com

\begin{abstract}
Introduction: Multilevel femur fracture is being encountered frequently than previously thought. The literature reports the incidence anywhere between 6-11\%. Management of these fractures in isolation are technically demanding, and presence of other bone or organ involvement complicates the scenario in achieving the optimal functional results. The use of a single implant provides the technically better overlapping fixation.

Methods: Our study represents a prospective cohort study in a 2-year period, between 2017 till 2019. Twenty-six patients with multilevel femur fractures being treated by a single step all in one interlocked cephalomedullary nail were included.

Results: In our study 16 patients $(88.8 \%)$ had been treated by Antegrade cephalomedullary reconstruction nail / proximal femoral nail. Healing of all the femoral fractures was without major complications or requirement of additional surgical procedures. In our study the femur fractures, on an average healed by 18 weeks.

Conclusion: For multilevel segmental femur fracture in young adults, stabilization using All-in-one device such as the intramedullary nail is recommended. Closed reduction with PFNA-long / Reconstruction nail is an effective treatment for long-segment femoral fracture with good strength in fixation, high rate of fracture union, and low rate of complications.
\end{abstract}

Keywords: Multilevel; femur fracture; intramedullary nail; segmental fracture

\section{INTRODUCTION}

$\mathrm{M}$ ultilevel femur fracture is being encountered frequently than previously thought. The literature reports the incidence anywhere between $6-11 \%$ (1). These fractures are common in young individuals involved in road traffic accidents (2). The ipsilateral multilevel femur fractures are high energy impact injuries and usually associated with polyskeletal or other organ injuries (3). Management of these fractures in isolation are technically demanding, and presence of other bone or organ involvement complicates the scenario in achieving the optimal functional results. Thereby increases morbidity in these young patients. There are many studies in the literature which deal with the treatment of these multilevel femur fractures using more than one region specific implant. This method of fixation though theoretically stabilizes individual fracture biomechanically with ideal implant, the use of non-overlapping implants in a single bone sometimes leads to implant failure, wound healing complications, prolonged operating time, excessive blood loss and decreased functional outcome in the long run. Intramedullary Femoral nail using intramedullary fixation technique for these multilevel femur fractures provides many advantages. The use of an All in one implant for these multilevel fractures significantly reduces the problems associated with use of more than one implant in these complex femoral fractures. The use of a single implant provides the technically better overlapping fixation. Single implant intramedullary nailing technique preserves the biology of the bone and hence healing occurs in a more desired way. This also reduces the surgical time and complications significantly.

\section{MATERIALS AND METHODS}

Our study represents a prospective cohort study in patients who sustained these complex multilevel fractures and being treated by a single step all in one interlocked cephalomedullary nail. In a 2-year period between 2017 till 2019 we treated 26 patients with multilevel femur fractures. All the patients in our study were young men with a median age of 28 years (range 21-35 years). We excluded patients who sustained open fractures and included 18 patients who had closed multilevel femur fracture and were within the age group of 18 till 40 years. All the patients were involved in a road traffic accident and presented to the hospital on the same day within 6 hours of injury. We recorded for all patients, demographic data, mechanism of injury, timing of stabilization, methods of reduction, type of stabilizing implants (AO PFN A2 / Reconstruction nail), time interval between trauma and surgery and follow up. 
In view of road traffic accident routine trauma series $\mathrm{x}$-ray and a routine Anteroposterior and Lateral view of the injured limbs were done. Patients who showed signs of head injury were subjected to a CT scan of the brain and a neurosurgeon's consultation was sought to R/O intracranial bleeding. In our study group we had 6 patients with associated injuries. All the patients were hemodynamically stabilized. We had $4(22 \%)$ patients who showed signs of compromised hemodynamic status. All these patients were treated by replacing the intravascular volume by IV fluids. None of the patients in our study group required blood transfusion. The patients were provided with adequate pain relief and then the limb was splinted using a mouldable long leg splint and shifted for radiological evaluation.

There were 2 patients (11\%) with ipsilateral tibia fracture, 3 patients $(16 \%)$ with pelvis and acetabular fracture and one patient $(5.5 \%)$ with clavicle fracture. The right lower limb was injured in 12 patients $(66.6 \%)$ and 06 patients $(33.3 \%)$ had involvement of their left lower limb. Fracture was classified according to AO/ASIF classification (4).

Standard postoperative care protocol was followed. Prophylactic antibiotic coverage was given till 48 hours with a third-generation IV cephalosporin. The wound was regularly checked on the $2^{\text {nd }}, 5^{\text {th }}$ and during the $12^{\text {th }}$ day for suture removal. No chemical thromboprophylaxis was given, though mechanical prophylaxis with a calf pump was used bilaterally. The patient was encouraged to do pelvic lift and right lower limb exercises actively. He was mobilized on the $3^{\text {rd }}$ day non weight bearing on the operated side with a walking frame support which was continued till 6 weeks. Partial weight bearing was started from the $6^{\text {th }}$ till $8^{\text {th }}$ week with walking aid. Full weight bearing was started at the end of the $8^{\text {th }}$ week.

Postoperative follow up was done till the patients showed signs of healing in the $\mathrm{x}$-rays. Routine follow up period was at 6 weeks, 10 weeks and 6 months. Healing was concluded with formation of bridging callus and bone trabeculae crossing the fracture line in at least three out of four cortices, as well as the absence of pain with full weight bearing.

\section{RESULTS}

In our study 16 patients $(88.8 \%)$ had been treated by Antegrade cephalomedullary reconstruction nail /proximal femoral nail. Two patients (12\%) had involvement of distal femur fracture which was stabilized by retrograde distal femoral nail. There were two intracapsular neck fractures (31-B2). Both femoral neck fractures had an oblique orientation. The neck fractures were treated by cervical bolts through the nail. All femoral shaft fractures were segmental middle third of the shaft: all were transverse (32-A3) and two were complex fractures (32-C1). One patient presented with a complex double segmental fracture of the shaft. There were two distal femoral fractures involving the condyle (33-B). One of the unicondylar fractures involved the medial condyle (33-B2) and the other was a fracture of the lateral condyle (33-B3; Table 1).

Table 1: Demographic data of the patients

\begin{tabular}{|c|c|}
\hline Number of patients & 18 \\
\hline Gender (M/F) & $18 / 0$ \\
\hline Mean Age in years & 28 \\
\hline Side (Right/Left) & $12 / 6$ \\
\hline $\begin{array}{c}\text { Fracture type (all fracture } \\
\text { are segmental with } \\
\text { following combinations) }\end{array}$ & $31 \mathrm{~B} 2-1,32 \mathrm{~A} 3-1$ \\
\hline $\begin{array}{c}\text { Time interval between } \\
\text { trauma and presentation to } \\
\text { ER (hours) }\end{array}$ & Mean - 5.8 \\
\hline Follow up in months & $26+/-08$ \\
\hline
\end{tabular}

There were two patients who had ipsilateral acetabular fracture one of which was treated by minimal invasive percutaneous screw fixation for the anterior column fracture and the other underwent plate fixation for posterior column fracture. One patient had sacroiliac joint disruption which was managed by screw fixation. The three patients with tibia fracture underwent closed interlocked intramedullary nailing. The average duration for the surgery was 1 'hour 50" minutes. The average blood loss during the surgery was estimated around $260 \mathrm{ml}$, no intraoperative blood transfusion was required.

Healing of all the femoral fractures was without major complications or requirement of additional surgical procedures. In our study the femur fractures, on an average healed by 18 weeks. Additionally, in one patient with shortening of the femur by $1.5 \mathrm{~cm}$, which was inevitable (due to a segmental comminution in the diaphyseal region), the fractures healed well. The femoral neck fractures united at an average of 14 weeks. There was no case of malunion in the diaphyseal fracture. All the fracture united without any rotational malalignment, one patient though had a varus malreduction of the proximal fragment. This patient had a subtrochanteric segmental shaft fracture treated by a proximal femoral nail (Fig. 1).

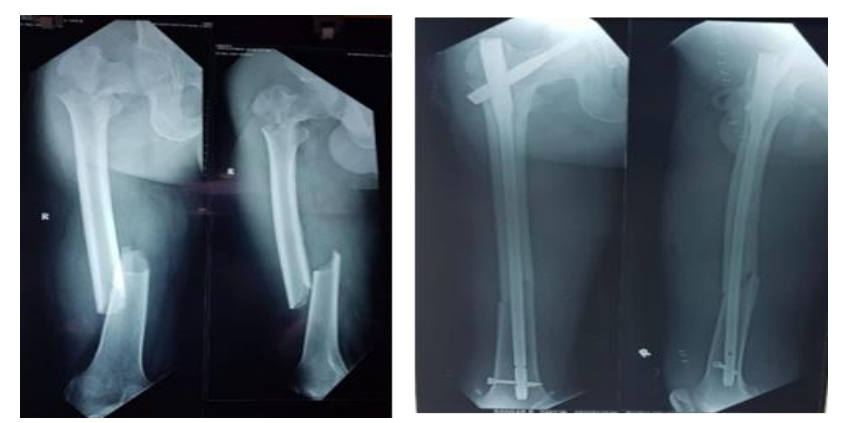

Fig. 1: Patient with subtrochanteric fracture treated by PFN A2 has varus malposition of the proximal fragment due to canal entry mismatch. 
Assessment of functional results demonstrated ROM of the hip joint with a mean of $110^{\circ}$ hip flexion and $\mathrm{ROM}$ of the knee joint with a mean of $120^{\circ}$ flexion. No extension deficit for both the hip and knee joints was observed.

\section{DISCUSSION}

Ipsilateral multilevel femoral fractures are high energy impact injuries. Multilevel fracture occurs due to axial loading of the femoral shaft, which usually occurs in the middle third of the femur followed by transmission of the force through the shaft longitudinally resulting in proximal femur fracture. There are many possible combinations of injuries that can occur in multilevel fractures of the femur. The most common injuries are femoral neck fractures followed by trochanter and distal femur as reported by Shuler et al., (5). In our study we had four patients $(22.2 \%)$ with proximal femur extracapsular fracture, two patients $(11 \%)$ had femoral neck fractures and the remaining ten patients (55\%) had segmental shaft fractures. One patient in our study had an undisplaced, basicervical neck fracture which was not evident in the preoperative $\mathrm{x}$-rays but was promptly recognized during the nailing, and was treated by using cervical bolts through the reconstruction nail.

The bending moment at the long segmental fractures is very large. This increases the stress on the implant making the choice of implant difficult and results in non-union and breakage of extramedullary fixation devices (6). Therefore, the best choice of internal fixation method for treating long-segmental fractures of the proximal and upper-middle part of the femur is still controversial (7). Regarding the surgical strategy of treating these patients it was important to choose an implant which will not interfere with the fixation of the concurrent fracture in the same bone. There are many reports of treating these injuries using more than one implant. DHS/Cervical cancellous screws/ PFN for the proximal fracture and a distal femoral nail/ distal femoral locking plates for distal femur fractures, used in combination have given mixed results. In the beginning, the idea of stabilizing the ipsilateral proximal (femoral neck or trochanter fracture) and the femoral shaft fracture using all-inone intramedullary implant was intriguing (8). But the biomechanical superiority of using a Intramedullary fixation to treat these fractures and the same implant to span the entire bone (All in One) offers the advantage of less soft tissue dissection, less operating time and blood loss. Initial reports have shown that the use of a cephalomedullary implant to address both fractures simultaneously led to significantly higher rate of mal-reduction of either of the fractures according to Bedi et al., (9).

Open methods of fracture fixation which strip the soft tissue attachments from the bone cause devascularisation of the middle segment (10). In our study all the patients were managed with a single closed Intramedullary nail to address the multilevel fractures of the femur. This significantly reduced the operating time, blood loss in our patients and preserves the vascularity of the floating segment. This closed method of treatment avoids healing complications. The new approach to manage ipsilateral femoral neck-shaft fractures by using reconstruction nails obtains relatively good clinical results (11). Anatomical reduction of the femoral neck fracture is key to prevent femoral neck non-union (12). In our study all the fractures united by 18 weeks and one patient with a displaced neck fracture showed signs of union at 14 weeks (Table 2).

Table 2: Blood loss, duration of surgery and healing time

\begin{tabular}{|c|c|}
\hline Average duration of surgery & 1 hour 50 minutes \\
\hline Operative blood loss & $260 \mathrm{ml}$ \\
\hline $\begin{array}{c}\text { Healing time of fracture } \\
\text { (average in weeks) }\end{array}$ & 18 \\
\hline $\begin{array}{c}\text { Implants used } \\
\text { (PFN/Reconstruction nail) }\end{array}$ & $11 / 7$ \\
\hline
\end{tabular}

Reduction of the proximal segment to align with the intermediate floating fragment and to gain perfect entry for the nail will always be challenging in these fractures. Malreduction of the proximal fragment before nail entry usually lead to varus malreduction of the fracture due to the nail canal mismatch that can occur due to the broader proximal segment in the reconstruction /proximal femoral nail. There are many techniques to aid in reduction of the proximal fragment. Some authors suggest application of an auxiliary plate osteosynthesis with monocortical screw fixation to increase rotational stability of the intermediate fracture fragment $(2,5)$. In our study we mostly use a Schanz screw in the subtrochanteric region to hold the fracture rotated and extended before the incision and nail entry. In spite of the above technique we had one case of varus angulation of the proximal fragment, but the angulation was minimal and the patient had uneventful fracture healing. In another patient the proximal femur fracture was held reduced with the segment beneath with a $3.5 \mathrm{~mm}$ Asian DCP to aid perfect entry into the canal (Fig. 2).

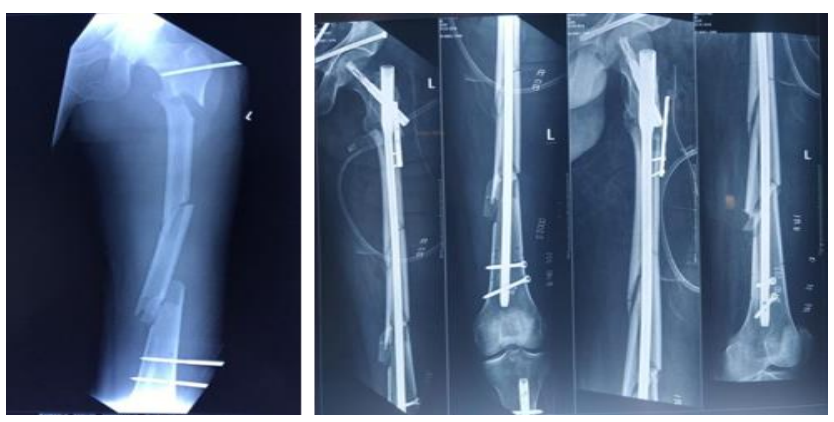

Fig. 2: Patient with double segment femur fracture treated by PFN A2. 3.5mm Asian DCP used to hold proximal fragment reduced to aid proper entry into canal. 
In eight patients we used Schanz screw as an indirect reduction tool in the intermediate/ distal fragment to hold it reduced before negotiating the guide wire into the distal fragment (Fig 3).
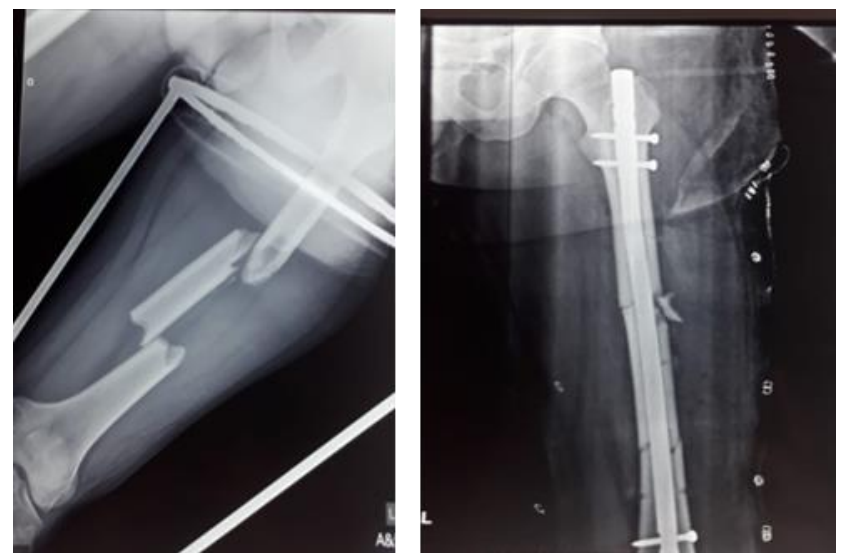

Fig. 3: Patient with segmental femur fracture which was reduced using a Schanz screw (seen as drill hole on the lateral cortex) to aid reduction before guide wire and nail entry.

In using more than one implant in treating these fractures, it is recommended to proceed with fracture stabilization from distally to proximally as recommend by Ruden et al., If the femoral neck fracture is displaced, the surgical strategy of choice is first to stabilize the shaft fracture followed by screw fixation of the proximal fracture. The stabilized shaft fracture facilitates reduction of the displaced proximal fracture. This surgical strategy facilitates fracture reduction and results in improved alignment of both fracture components (13). This sometimes requires an external fixator to hold the other fracture during fixation of fracture at another level in the same femur. In our study 17 patients underwent single stage implant fixation and this avoids the need for temporary fixation using an external fixator, thereby significantly reducing operating time. One patient in our study group had associated polyskeletal injury (ipsilateral tibia, acetabular and pelvic fracture). This patient had to be temporarily stabilized in an external fixator.

\section{CONCLUSION}

For multilevel segmental femur fracture in young adults, stabilization using All-in-one device such as the intramedullary nail is recommended. Fractures, which are extraarticular and localized very distally, are best treated with retrograde intramedullary nailing. Varus malposition of the proximal fragment can be avoided by reducing the fracture prior to making an entry for nail insertion. Closed reduction with PFNA-long / Reconstruction nail is an effective treatment for long-segment femoral fracture with good strength in fixation, high rate of fracture union, and low rate of complications.

\section{CONFLICT OF INTEREST}

Authors declare no conflict of interest.
1. Tsarouhas, A., Hantes, M. E., Karachalios, T., Bargiotas, K., Malizos, K. N. Reconstruction nailing for ipsilateral femoral neck and shaft fractures. Strategies Trauma Limb Reconstr. 2011; (6): 69-75.

2. Gadegone, W., Lokhande, V., Salphale, Y., Ramteke, A. Long proximal femoral nail in ipsilateral fractures proximal femur and shaft of femur. Indian J Orthop.2013; (47), 272277.

3. Enninghorst, N., McDougall, D., Evans, J. A., Sisak, K., Balogh, Z. J. Population-based epidemiology of femur shaft fractures. J Trauma Acute Care Surg. 2013, 74: 1516-1520.

4. Müller, M. E., Nazarian, S., Koch, P., Schatzker, J. The comprehensive classification of fractures of long bones. Berlin New York: Springer; 1990.

5. Shuler, T. E., Gruen, G. S., DiTano, O., Riemer, B. L. Ipsilateral proximal and shaft femoral fractures: spectrum of injury involving the femoral neck. Injury. 1997; 28(4): 293 297.

6. Cheng, Y. X., Sheng, X., Optimal surgical methods to treat intertrochanteric fracture: a Bayesian network meta-analysis based on 36 randomized controlled trials. Journal of Orthopaedic surgery and research. 2020; 15: 402.

7. Mereddy, P., Kamath, S., Ramakrishnan, M., Malik, H., Donnachie, N. The AO/ASIF proximal femoral nail antirotation (PFNA): a new design for the treatment of unstable proximal femoral fractures. Injury. 2009; 40: 428432.

8. Hoover, G. K., Browner, B. D., Cole, J. D., Comstock, C. P., Cotler, H. B. Initial experience with a second generation locking femoral nail: the Russel-Taylor reconstruction nail. Contemp Orthop. 1991; 23(3): 199-208.

9. Bedi, A., Karunakar, M. A., Caron, T., Sanders, R. W., Haidukewych, G. J. Accuracy of reduction of ipsilateral femoral neck and shaft fractures--an analysis of various internal fixation strategies. J Orthop Trauma. 2009 Apr; 23(4): 249-253.

10. Howe, T. S. Double level fractures of the femur treated with closed intramedullary nailing. Ann Acad Med Singapore. 1998 Mar; 27(2): 188-191

11. Lin, S. H., Lo, C. W., Cheng, S. C., Kuo, M. Y., Chin, L. S. Use of reconstruction nails to manage ipsilateral displaced femoral neck-shaft fractures: assessment of a new approach. J Orthop Surg (Hong Kong). 2002 Dec; 10(2): 185-193.

12. Gary, J. L., Taksali, S., Reinert, C. M., Starr, A. J. Ipsilateral femoral shaft and neck fractures: are cephalomedullary nails appropriate? J Surg Orthop Adv. 2011 Summer; 20(2): 122-125.

13. Tsai, M. C., Wu, C. C., Hsiao, C. W., Huang, J. W., Kao, H. K., Hsu, Y. T. Reconstruction intramedullary nailing for ipsilateral femoral neck and shaft fractures: main factors determining prognosis. Chang Gung Med J. 2009; 32(5): 563-573.

\section{REFERENCES}

